\title{
Multi-layer coating development for XEUS
}

D. H. Lumb, M. Bavdaz, F. E. Christensen, A. Dariel, P. Hoghoj, et al.

D. H. Lumb, M. Bavdaz, F. E. Christensen, A. Dariel, P. Hoghoj, C. P. Jensen, M. Krumrey, K. K. Madsen, E. Ziegler, B. Albertin, S. Hedacq, M. Collon, E.-J. Buis, "Multi-layer coating development for XEUS," Proc. SPIE 6266, Space Telescopes and Instrumentation II: Ultraviolet to Gamma Ray, 626614 (13 June 2006); doi: 10.1117/12.669946

SPIE Event: SPIE Astronomical Telescopes + Instrumentation, 2006, Orlando, Florida, United States 


\title{
Multi-Layer Coating Development for XEUS
}

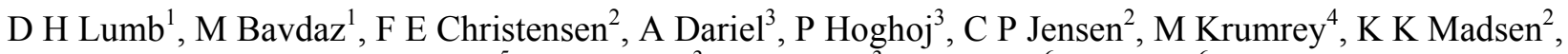

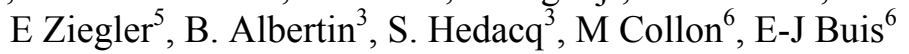 \\ 1) Advanced Concepts and Science Payloads Office, European Space Agency, ESTEC, Postbus 299, \\ 2200AG Noordwijk, Netherlands \\ 2) Danish National Space Centre, DK-2100 Copenhagen Ø Juliane Maries Vej 30, Denmark \\ 3) Xenocs, 19, rue François Blumet, F-38360 Sassenage, France \\ 4) Physikalisch-Technische Bundesanstalt, Abbestr. 2-12, 10587, Berlin, Germany, \\ 5) ESRF,BP220, 38043 Grenoble, France \\ 6) Cosine Research, NielsBohrweg 11, 2333CA Leiden, Netherlands
}

\begin{abstract}
Graded depth multi-layer coatings have the potential to optimise the performance of X-ray reflective surfaces for improved energy response. A study of deposition techniques on silicon substrates representative of the XEUS High Performance Pore Optics (HPO) technology has been carried out. Measurements at synchrotron radiation facilities have been used to confirm the excellent performance improvements achievable with $\mathrm{Mo} / \mathrm{Si}$ and $\mathrm{W} / \mathrm{Si}$ multilayers. Future activities that will be necessary to implement such coatings in the HPO assembly sequence are highlighted. Further coating developments that may allow an optimisiation of the XEUS effective area in light of potential changes to science requirements and telescope configurations are also identified. Finally an initial measurement of effects of radiation damage within the multilayers is reported.
\end{abstract}

Keywords: X-ray astronomy, X-ray optics, telescopes, coatings

\section{INTRODUCTION}

Elsewhere in this volume ${ }^{1,2,3}$, the design of the optics for the X-Ray Early Universe Spectroscopy (XEUS) mission is presented. The heart of the optics is a novel silicon plate technology that allows very close stacking of reflector plates to provide a conical approximation to the Wolter-1 grazing incidence telescope design. This allows a very lightweight implementation, and with a long focal length the angular resolution is determined by simple geometry, where for XEUS the goal is 2 arcsec FWHM. The primary scientific motivation is the study of the early Universe, and through the process of cosmological red shift, this implies most photons of interest will be focused at energies $<2 \mathrm{keV}$. As reported in Bavdaz et $\mathrm{al}^{4}$ the XEUS mission concept has been studied with different launch options. Studies carried out jointly with NASA to consider use of a Delta IV Heavy vehicle have been abandoned in light of the uncertainties in the Beyond Einstein programme. Recent mission study activities have concerned the trade-off of deployable and non-deployed telescope designs that can be accommodated within the constraints of a single Ariane V launch.

The constraints of both mass and fairing envelope are still being considered, so that detailed designs are yet to be completed. The effective area that is likely to result from these redesigns may be restricted at the lowest energies by the launcher envelope, and a central spacecraft sub-system hub. In order to preserve the maximum effective area at low energies, which is required by the primary science goals, reducing the focal length and increasing reflection angles allows a more efficient utilisation of frontal collecting area. In consequence the lower limit of grazing angle is changed with a significant impact on the ability to achieve the required reflectivity at the highest energies, so that the ancillary science goal of providing a hard X-ray imaging capability is also affected.

Pending the completion of design studies, and parallel reassessment of science requirements, we continue to explore additional benefits to tuning the energy dependent effective area by employing multilayer coatings. In this paper we describe results of a development programme for deposition of graded depth multilayer coatings on silicon samples. We describe some design activities carried out in the context of the original XEUS configuration, and how this results in the

Space Telescopes and Instrumentation II: Ultraviolet to Gamma Ray, edited by

Martin J. L. Turner, Günther Hasinger, Proc. of SPIE Vol. 6266, 626614, (2006)

0277-786X/06/\$15 - doi: 10.1117/12.669946

Proc. of SPIE Vol. 6266 626614-1 
selection of some specific multilayer lay-ups to be explored. We describe measurements made at synchrotron facilities on a number of these multilayer samples. Future work to optimise reflectivity at different energies is proposed, and practical considerations for implementing multilayers within the silicon stacking process for the XEUS optics are described.

\section{XEUS BASELINE ACTIVITY}

Design work on multi-layer coatings started some years ago, when the capability for hard X-ray astronomy with a $50 \mathrm{~m}$ focal length telescope was recognised. In this case the concept for an initial free-flying XEUS considered the launch into an ISS fellow-traveler orbit of a pair of satellites comprising a Mirror Spacecraft (MSC) and a Detector Spacecraft (DSC) flying in formation. In the initial phase the telescope accommodated on the MSC had optics segments arranged in radii from $\sim 0.7$ to $1.95 \mathrm{~m}$, and with the $50 \mathrm{~m}$ focal length, this allowed for grazing angles of reflection from $\sim 3$ to 10 mrads. These parameters were assumed in studying the coating designs.

The selection of the best absorber/spacer pair for XEUS multilayers began with the analysis of the system requirements, followed with the selection of spacer candidates with minimum absorption and then the absorber candidates with maximum contrast. Finally the best pair in term of optical performance, as well as physical and chemical compatibility was picked. A firm requirement to be able to measure Ti X-rays of $78 \mathrm{keV}$ energy from supernovae was used to define the upper energy range so that the XEUS-1 telescope should be able to reflect $\mathrm{x}$-rays up to 70 to $80 \mathrm{keV}$ in the inner radii. The higher the grazing angle the more difficult it is to reflect x-rays of higher energy, even with multilayer designs with extremely thin periods (at least $25 \AA$ ) and as many as 1000 layers, so the design considered optimizing for 40 to 50 $\mathrm{keV}$ in the middle radii, and only up to $20 \mathrm{keV}$ in the outer radii. These coarse ranges were further broken down into a total of $\sim 30$ mirror groups (radii) to allow optimisation of material selection. For the first absorber selection we limited our calculations to the Si spacer because the results are very similar for all spacer materials.

The Figure Of Merit (FOM) established was the sum of the integrated throughput of the mirror shells in a given mirror group over the energy band and field of view of the telescope. The calculation of the FOM requires the geometry of the telescope optics, weighting functions for spectral and angular response and the matrix of multilayer reflectivity vs. energy and incidence angle. The integration over off-axis angles was performed for an assumed 5-arcmin field of view of the high energy XEUS detector. Finally the FOM was then obtained by summing over the mirror shells in the mirror group and integrating over the energy band foreseen for the mirror group, and allowing the higher energies to be weighted by the energy weighting function. In the non analytical approach to optimising the multilayer coating in each mirror group we have adopted a power law progression of the d-spacings in the stack, which covers a wide performance space and thus globally provide optimised designs even for rather different energy- and angular- weighting functions as the FOM allows for.

The power law progression is defined by : $d(i)=a /(i-b)^{c}$ where the first bilayer $(i=1)$ is the bilayer in the top of the stack and the last bilayer $(\mathrm{i}=\mathrm{N})$ is the bilayer next to the substrate. We have further assumed that the ratio between the thickness of the heavy element and the bilayer thickness of every bilayer is a constant $-\gamma$. Thus a complete description of a given coating is specified by the five parameters ; $\mathrm{a}, \mathrm{b}, \mathrm{c}, \mathrm{N}$ and $\gamma$. Alternatively one can specify the minimum dspacing $d_{\min }=d(N)$, the maximum d-spacing $d_{\max }=d(1), c, N$ and $\gamma$. A complete optimisation would entail walking through all of the available parameter space for each of the five parameters. This is, however, an extremely time consuming process and it is not necessary as it is straightforward to set the limits $d_{\min }$ and $d_{\max }$ for each mirror group based on practical coating considerations and global considerations on desirable energy band for a given mirror group. Thus the coating optimisation is reduced to varying $c, \gamma$ and $\mathrm{N}$ for selected $\mathrm{d}_{\min }$ and $\mathrm{d}_{\max }$ for each mirror group so as to maximise the FOM for that mirror group.

To establish designs for initial coating tests we have chosen to let the inner mirror groups be composed of Pt/C which allows for throughput up to the Pt-K edge at $78.4 \mathrm{keV}$. The mirror groups in the middle of the radius range we have chosen to be W/Si until we reach a radius where it is no longer feasible to deposit the small d-spacings required to get throughput above $20-30 \mathrm{keV}$. From that radius and out to the max radius of $1.95 \mathrm{~m}$ we have chosen to use $\mathrm{Mo} / \mathrm{Si}$ as this is a very efficient reflector at energies just below $20 \mathrm{keV}$ due to the $\mathrm{K}$-edge of Mo at $20 \mathrm{keV}$. With these choices we get the initial coating designs for a multilayer set that can be deposited and performance tested in X-rays. 


\section{ROUGHNESS REDUCTION}

The most critical parameter to produce a high performance x-ray supermirror is the interface roughness of the coatings. A low roughness level requires of course using very smooth substrates; in addition deposition process improvements can lead to lower roughness.

For the Mo/Si coatings we investigated the use of barrier layers to reduce the multilayer roughness, initially with trials on periodic coatings before replicating the nominal graded density $\mathrm{Mo} / \mathrm{Si}$ test design. Based on previous studies on coatings for $13.5 \mathrm{~nm}$ wavelength applications, a proprietary interface was attempted, with a $0.4 \mathrm{~nm}$ layer replacing a corresponding depth of Mo on each Mo over Si interface. Also the use of C atoms was investigated, where these small atoms are expected to insert efficiently into the previous layers. $0.5 \mathrm{~nm}$ of $\mathrm{C}$ was used to replace a corresponding depth of Mo on each Mo over Si interface.

An alternate approach for the W/Si stacks involves the use of modified sputtering gas during the Si deposition process. Again a study was conducted on periodic and graded W/Si stacks using different concentrations of gas.

For both techniques, an assessment of the performance improvement was made initially by reflectivity measurements at $8 \mathrm{keV}$. Subsequently, and in order to demonstrate performance directly in the energy range of interest, the reflectivity was measured at energies in the range $20-40 \mathrm{keV}$ at synchrotron facilities. Based on fitting with the IMD code ${ }^{5}$, the roughness of the interfaces was estimated

\section{MEASUREMENTS WITH SYNCHROTRON RADIATION}

\subsection{BESSY}

The W/Si multilayer mirrors were investigated at the BAMline, a wavelength-shifter beamline at BESSY which is jointly operated by the German Institute for materials research and testing (BAM) and the Physikalisch-Technische Bundesanstalt (PTB). This beamline is equipped with a double-multilayer monochromator (DMM) and a double-crystal monochromator (DCM). Both monochromators can be operated in series to combine the high spectral resolving power of the DCM with the efficient higher order suppression of the $\mathrm{DMM}^{6}$. While the photon energy range from $8 \mathrm{keV}$ to 60 $\mathrm{keV}$ is accessible without crystal change, the results presented were obtained at a fixed energy of $30 \mathrm{keV}$. The samples were installed in the center of rotation of a six-axis diffractometer by placing them on a flat silicon crystal without clamping it order to avoid a deformation. However, it turned out that the substrates had an intrinsic curvature possibly due to the deposition process. Therefore the true angle of reflectance was determined by using an X-ray sensitive CCDcamera system. From the distance between camera and sample and the pixel size of $23.5 \mu \mathrm{m}$, the angle setting has been verified.

\subsection{ESRF}

The measurements of the Mo/Si and NiV/C multilayer mirrors were conducted at the ESRF BM05 station ${ }^{7}$. To minimise uncertainties caused by sample curvature, a vacuum chuck was used for sample mounting. Unfortunately the nominal vertical beam reflectometer that is preferred for this sample characterisation was found not to be compatible with the large mass loading on the sample stage. Therefore a horizontal reflectometer configuration was adopted. That had a disadvantage of being unable to utilise the natural width of the synchrotron beam, so that the horizontal divergence has less dynamic range for the faintest scatter measurements. This beam configuration was essentially adapted from the 3axis diffractometer table to provide $\theta / 2 \theta$ scanning capability using the central goniometer mechanisms. The vacuum chuck was mounted on the goniometer head. The chuck has nominal flatness within 1 micron in the preferred mounting direction, but in order to accommodate the envelope of the chuck and its mounting bracket onto the goniometer, it had to be placed in the orthogonal direction, which has worse (up to 5 microns) flatness specification. Mo/Si samples were measured at energies of 18, 28 and $40 \mathrm{keV}$, with reflectivity and transverse scans. 


\section{RESULTS}

\section{1. $\mathrm{W} / \mathrm{Si}$}

The test design for W/Si employed 291 bi-layers, with $d_{\min }$ and $d_{\max } 2.4$ and $20.1 \mathrm{~nm}$ respectively. The $\mathrm{c}$ and $\gamma$ factors were 0.19 and 0.36 . In addition to the baseline design, two samples were fabricated in which different amounts of admix concentrations were included in the silicon sputtering process. The multilayers were deposited on $10 \mathrm{~mm} \times 100 \mathrm{~mm}$ silicon strips obtained from the same process as wafers used for the XEUS prototype optics.

Measurements of reflectivity at $8 \mathrm{keV}$ for precursor periodic coatings (d-spacing $4 \mathrm{~nm}$ ) were made. These established that the $\mathrm{Si} / \mathrm{W}$ interface roughness was constant at $\sim 0.3 \mathrm{~nm}$, while the W/Si interface roughness significantly depended on the content of the sputtering gas. The roughness inferred by comparing reflectivity curves with IMD predictions indicated a roughness from $0.5-0.8 \mathrm{~nm}$ at nominal gas mixture, improving to $0.3-0.4 \mathrm{~nm}$ for the modified gas.

The reflectivity of the graded depth multilayer coatings was measured at BESSY at an energy of $30 \mathrm{keV}$. While the effect of the curvature of the samples was accommodated by the procedure described in section 4.1, the varying curvature between different samples was suspected to lead to different beam projection at the smallest graze angles. An empirical renormalisation has been applied for graze angles $<0.2^{\circ}$, and this remains uncertain. The resulting reflectivity curves are plotted in Figure 1. The best fits to bi-layers parameters were very close for all three samples, and the major difference evidenced by a change in reflectivity, especially in the range $>0.5^{\circ}$. The total roughness inferred for the $\mathrm{W} / \mathrm{Si}$ interface in each case was consistent with the values determined from the periodic coatings.

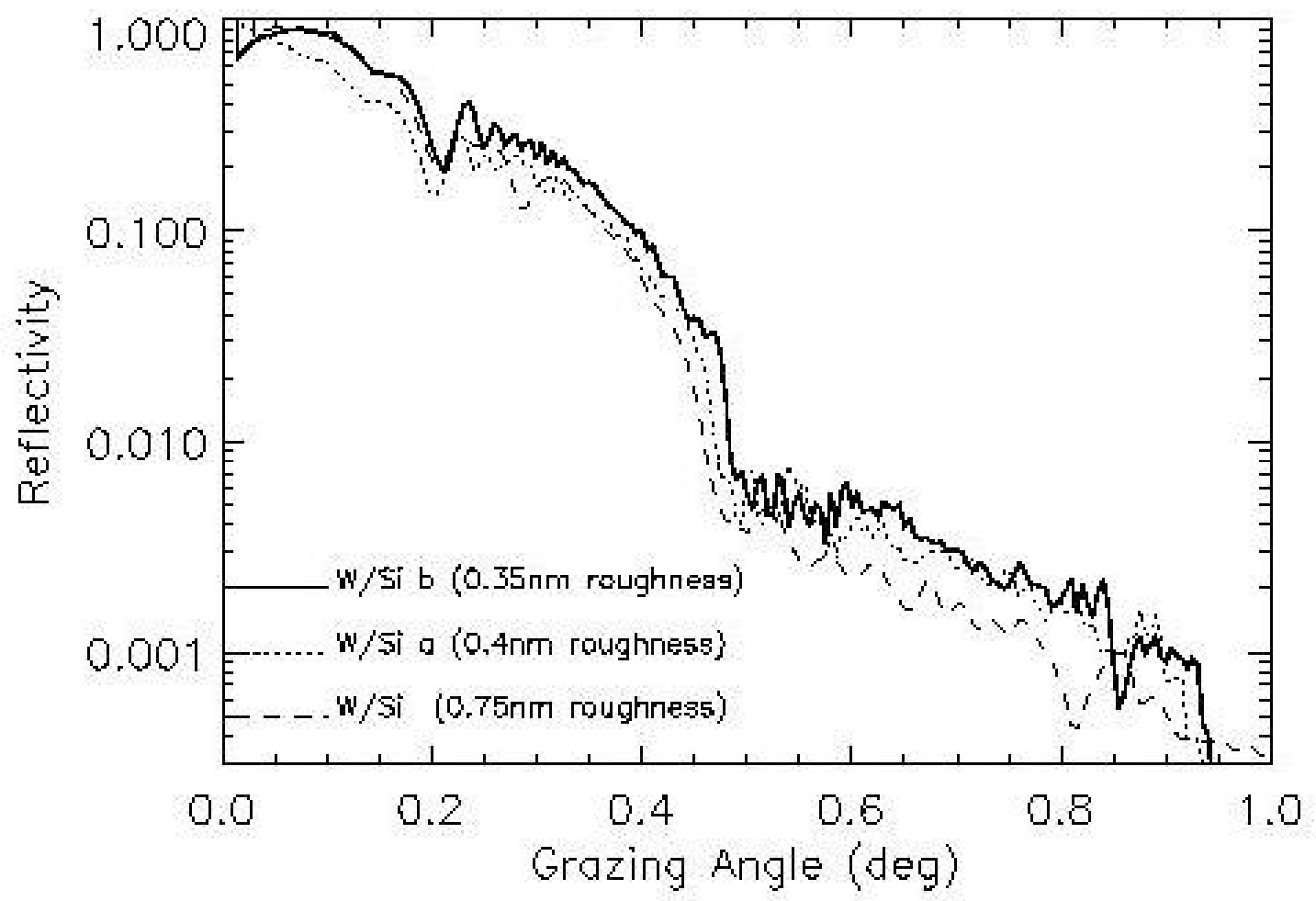

Figure 1 Comparison of $30 \mathrm{keV}$ reflectivity for three samples of W-Si graded density multilayer with different gas concentrations during sputtering 
The data are consistent with roughness of substrate and $\mathrm{Si} / \mathrm{W}$ interface of $0.25-0.3 \mathrm{~nm}$, and with the high admix to the Ar sputtering medium a similar roughness is achieved in the $\mathrm{W} / \mathrm{Si}$ indicating that the process can be reliably extrapolated for near theoretical performance with the ultimate roughness.

\section{2. $\mathrm{Mo} / \mathrm{Si}$}

The test design for Mo/Si employed 291 bi-layers, with $\mathrm{d}_{\min }$ and $\mathrm{d}_{\max } 3.3$ and $27.1 \mathrm{~nm}$ respectively. The $\mathrm{c}$ and $\gamma$ factors were 0.21 and 0.39 . In addition to the baseline design, two samples were fabricated in which different barrier layers were employed. The multilayers were deposited on identical silicon strips as the W/Si samples. Measurements of reflectivity at $8 \mathrm{keV}$ for precursor periodic coatings (d-spacing $4 \mathrm{~nm}$ ) were made. These established that the Si interface roughness was reduced with the introduction of barrier layer.

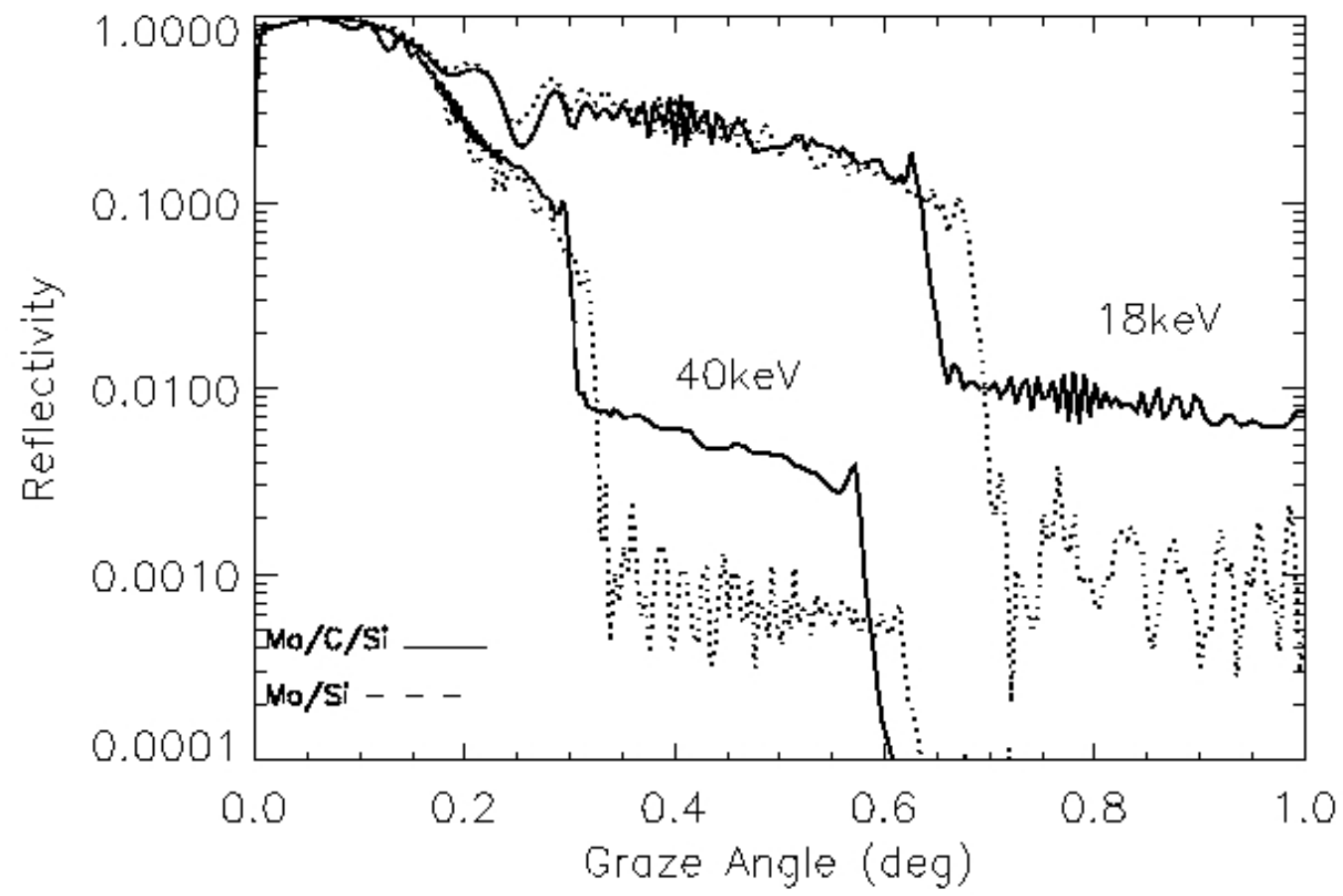

Figure $2 \mathrm{Mo} / \mathrm{Si}$ multilayer reflectivity measured at ESRF at 18 and $40 \mathrm{keV}$ with $(-)$ and without (- - -) a carbon barrier layer of 5 Angstroms

The reflectivity of the graded depth multilayer coatings was measured with the ESRF reflectometer at energies of 18, 28 and $40 \mathrm{keV}$. A selection of resulting reflectivity curves are plotted in Figure 2. Comparing reflectivities at angles greater than the first Bragg peak reveals an improved roughness value obtained with the $\mathrm{C}$ introduction, of from $\sim 0.55$ $\mathrm{nm}$ to $\sim 0.3 \mathrm{~nm}$.

\section{FURTHER WORK}

\subsection{High Energy Extension}

With the evolution in XEUS telescope envelope, preserving the hard X-ray performance is at a premium. Maximising this performance requires the manufacturing of the thinnest $\mathrm{d}$-spacings possible together with the lowest possible interface roughness. Elsewhere in this volume are reported similar developments but targeted at energy ranges $>100$ $\mathrm{keV}^{8}$. Using a NiV alloy, we investigated the use of Si and $\mathrm{C}$ spacers. Reflectivity of a $(\mathrm{NiV} / \mathrm{C})$ multilayer was higher than for a $(\mathrm{NiV} / \mathrm{Si})$ multilayer. Measurement of the reflectivity at $40 \mathrm{keV}$ for the $\mathrm{NiV} / \mathrm{C}$ multilayer is shown in Figure 3, in comparison with the $\mathrm{Mo} / \mathrm{C} / \mathrm{Si}$ coating as reference. The multilayer stack comprised equal thicknesses of $\mathrm{NiV}$ and amorphous carbon, with 600 bi-layers ranging from 12 to $1.2 \mathrm{~nm}$ in thickness. The inferred roughness is about $0.3 \mathrm{~nm}$. 
Figure 3 qualitatively compares the resulting effective area of the XEUS optics with the two coatings. In this case we take a worst case assumption that the inner radius is limited by an Ariane V launch adaptor so that $\mathrm{R}>0.6 \mathrm{~m}$
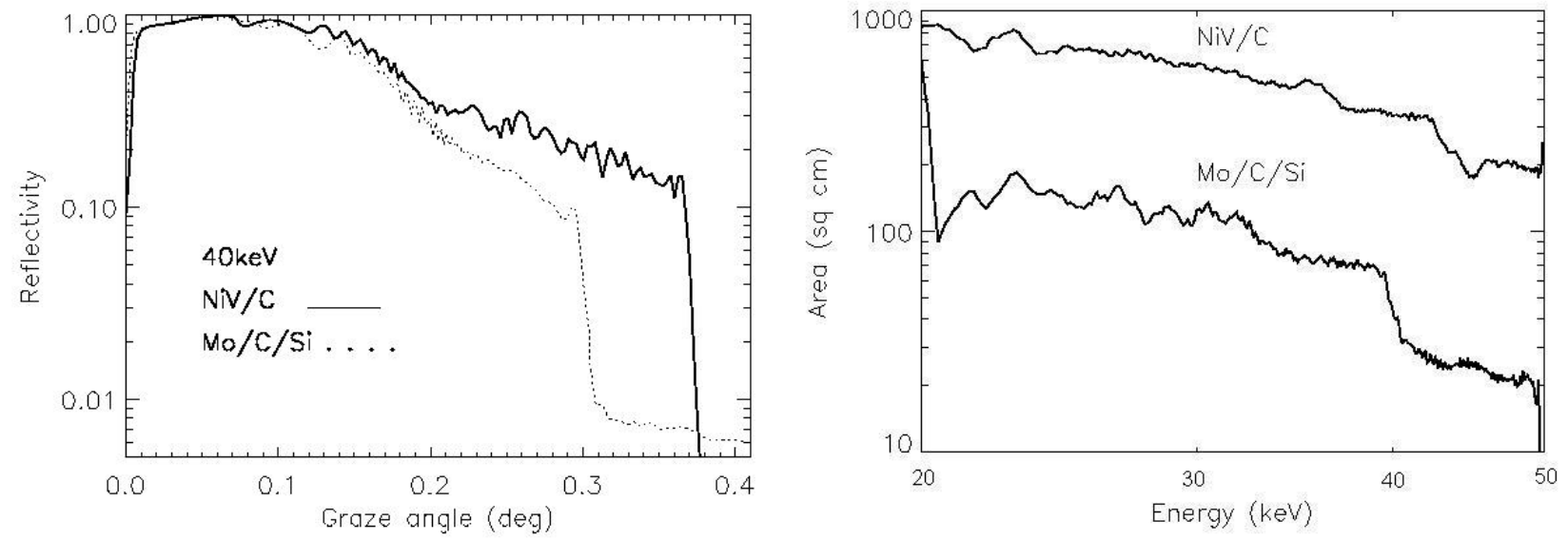

Figure 3 Improved X-ray reflectivity of NiV/C multilayer, compared with Mo/C/Si. (Left) Reflectivity measured at ESRF versus graze angle at $40 \mathrm{keV}$. (Right) Calculated effective area of XEUS telescope with restricted inner radius $>0.6 \mathrm{~m}$, using the optical constants derived from $40 \mathrm{keV}$ data

\subsection{Soft X-ray efficiency}

Since the most important performance requirement for the XEUS science goals remains the effective area at $1 \mathrm{keV}$, the reduction in envelope of the optics can be an important factor to be mitigated. Multilayer mirrors can be also used to enhance the effective area in this low energy band compared with single-layer reflectors' performance, as proposed by Pareschi ${ }^{9}$. A gain in reflectivity (in a restricted energy range between $\sim 0.5$ and $4 \mathrm{keV}$ ) can be achieved by using a low density material as the first external layer of the multilayer stack. This reduces the photoelectric absorption effect where the mirror acts in total external reflection regime. We have considered Carbon as the most useful material for this application.

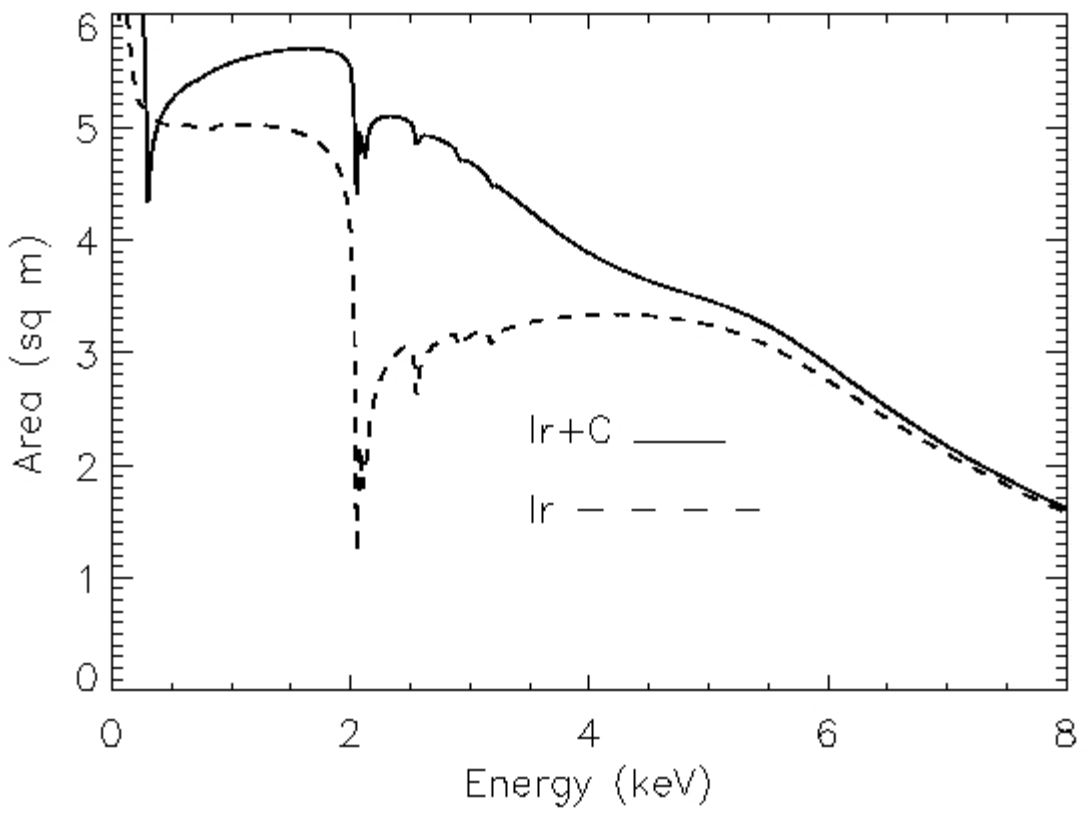

Figure 4 Comparison of XEUS effective areas with an Iridium reflective coating, and Iridium with a 100 Angstrom carbon overcoat. A roughness of $0.3 \mathrm{~nm}$ is assumed for this calculation 
For example Figure 4 compares the effective area attainable with a XEUS telescope design implemented with a single Ir layer, and a Ir layer over-coated with carbon. In this design we consider the geometric area of XEUS descoped to a restricted non-deployable envelope and examine the benefit that the $\mathrm{C}$-overcoat brings to regaining as much effective area at $1 \mathrm{keV}$ as possible; $20 \%$ improvement appears achievable.

\subsection{Radiation Damage}

Recalling that use of CCDs in space-borne instruments was initially compromised by unexpected effects of displacement damage in silicon, we judged it important at an early phase of the studies, to consider whether exposure to irradiation might have an effect on the integrity of the very thin multi-layers. Initial modeling with the TRIM code ${ }^{10}$ suggests the effect of atomic displacements will not likely modify the layered structure. Nevertheless we have made a first qualitative verification by exposing some test multilayers at a proton beam. Samples were irradiated with $\sim 184 \mathrm{MeV}$ protons to a fluence of $10^{12}$ protons $\mathrm{cm}^{-2}$. This is comparable with doses expected from large solar flares. Figure 5 presents the reflectivity measured at $8 \mathrm{keV}$ before and after irradiation, on a sample fabricated with W/SiC layers. No gross change in the reflectivity edge and oscillations has occurred indicating no gross structural changes in the multilayers. In addition, to within the measurement accuracy there is no significant reflectivity loss.

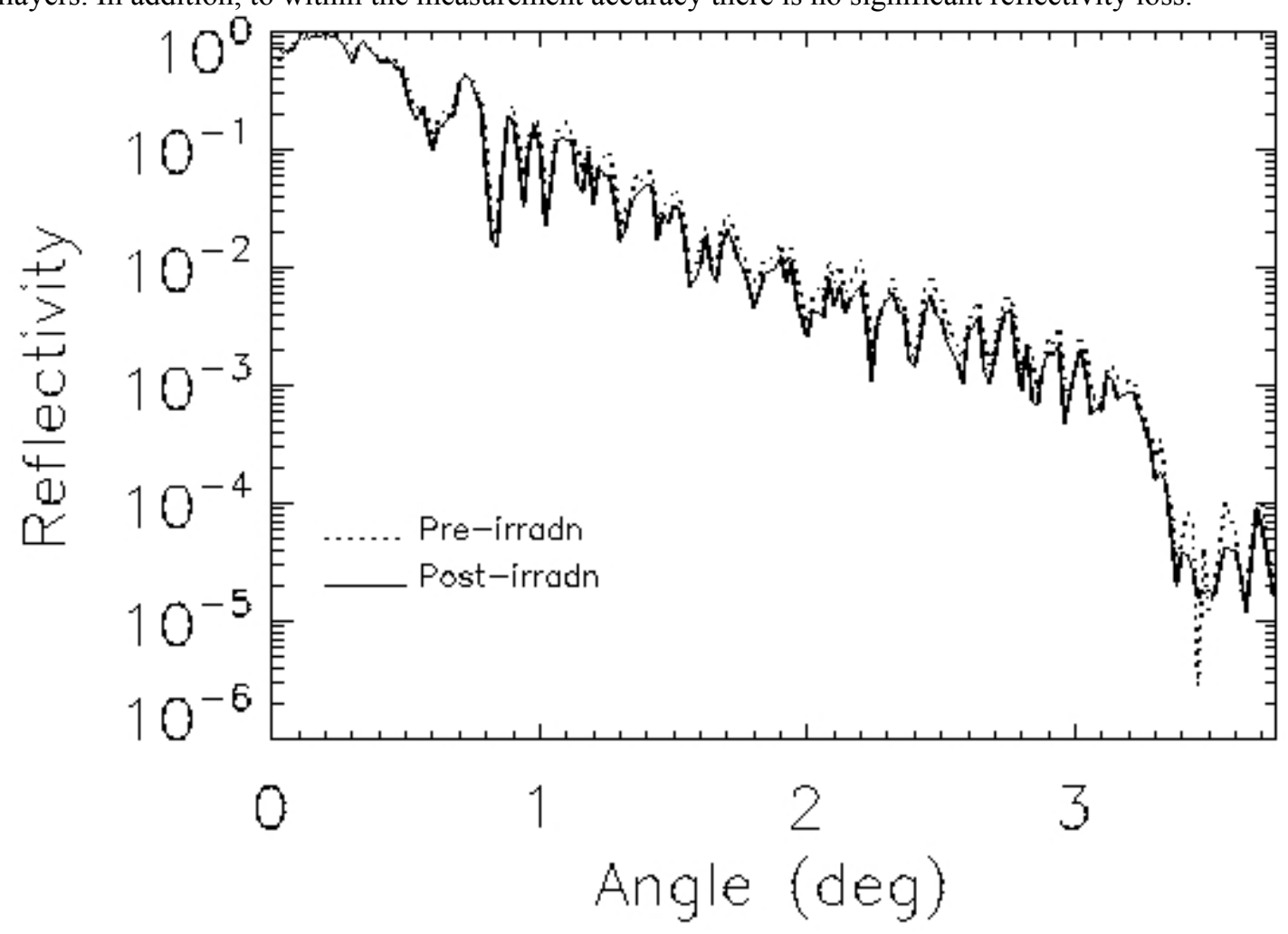

Figure 5 Comparison of reflectivity at $8 \mathrm{keV}$ for a $\mathrm{W} / \mathrm{SiC}$ multilayer measured pre- and post-irradiation to $184 \mathrm{MeV}$ protons. The fluence was $10^{12}$ protons $\mathrm{cm}^{-2}$

\section{MANUFACTURING PROCESS}

The High-performance Pore Optics (HPO) fabrication process involves precision alignment of the ribs of a structured silicon plate to the next (Fig. 6). The ribs of one plate are placed onto the high quality reflecting surface of the following one, and can, for example, permanently be attached via a hydrophyllic bonding process. The very small pores $(\sim 700$ $\mu \mathrm{m})$ do not facilitate metallic coating after assembly via CVD-like techniques, and are wholly unsuitable for bilayer depositions. Therefore the approach should be to coat the silicon reflecting surface as a precursor to the bonding. 


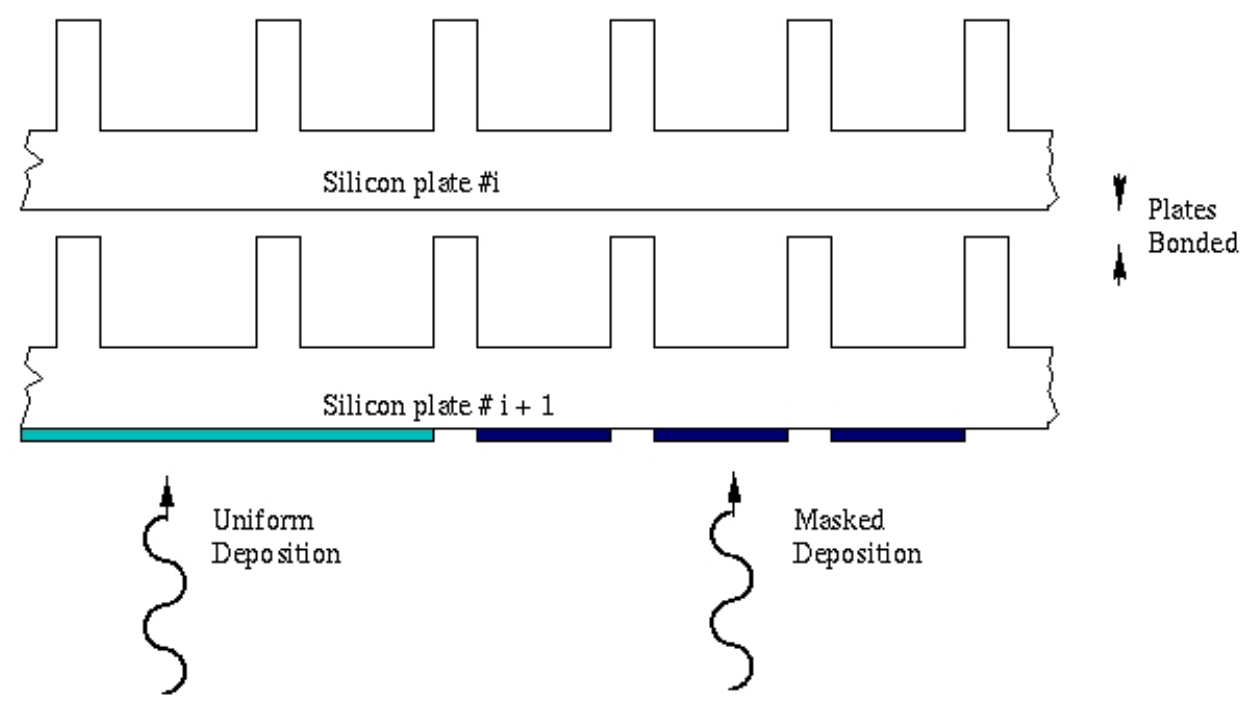

Figure 6 The coating process for the silicon surface eventually relies on the type of bonding adopted. In the simplest form a uniform coating can be applied to the whole plate surface, but then the final layer must be suitably finished for (e.g.) a hydrophilic bonding to the subsequent plate (LEFT). Alternatively the silicon surface to be coated must be lithographically masked to keep the silicon surfaces for bonding clear of deposited material.

It can be seen there are several development steps to be solved in this approach. Selection of a coating for optimum reflectivity must also respect the handling constraints. A minimum of handling steps must be maintained in the coating and/or masking process to ensure that no significant build up of particulate contamination can occur that impedes the subsequent bonding process and distorts the plate figure. The masking process, and thus the location of coated and uncoated areas, has to be sufficiently accurate to not impact on the HPO assembly process. A chosen coating has to be compatible with chemical processes required to prepare the silicon plates for bonding. In the case of a uniform coating approach the bond strengths and therefore the stiffness of the HPO structure have to be analyzed. This also applies to eventual adhesion layers, required to achieve e.g. Au coatings. Thermal treatment of HPOs will have an impact on the manufacturability of multi-layer coatings.

To address some of these questions already at an early stage a first set of masked coated silicon plates ( $\mathrm{Au} / \mathrm{Ti}, \mathrm{Pt}, \mathrm{W})$ have been produced in the current XEUS optics development program. These plates are currently being analysed in terms of reflectivity, surface roughness and coating adhesion strength. Different coating processes are being studied, and special transportation frames, suitable for both the coating and production facilities have been developed.

\section{CONCLUSION}

We have demonstrated the principle that multilayer coatings can be deposited on silicon wafer material representative of the XEUS mirror substrates. The initial surface roughness of the wafers is $\sim 0.2-0.3 \mathrm{~nm}$, and the interface roughness can be maintained at such levels through the growth process. This interface roughness has been confirmed by measurements of reflectivity at synchrotron facilities, and is crucial in maintaining performance at hard X-ray energies. It has been demonstrated that the roughness can be improved by use of barrier layers or improved sputtering processes. This gives confidence that the energy response of the XEUS telescope can be eventually optimised according to science goals and requirements, or to accommodate modification of the optics envelope. Progress towards implementing techniques that allow the coatings to be included in the silicon wafer stacking process has been made. 


\section{REFERENCES}

1. The XEUS X-ray Telescope, M. Bavdaz, D. H. Lumb, P. A. Gondoin, T. van der Laan, Proc SPIE 6266 this volume

2. The XEUS mission, A. N. Parmar, M. Arnaud, X. Barcons, J. A. Bleeker, G. Hasinger, H. Inoue, G. G. C. Palumbo, M. J. L. Turner, Proc SPIE 6266 this volume

3. Metrology, integration, and performance verification of silicon pore optics in Wolter-I configuration, M. Collon, S. Kraft, M. W. Beijersbergen, C. van Baren, M. Bavdaz, Proc SPIE 6266 this volume

4. M. Bavdaz, D. H. Lumb, A Peacock, M. W. Beijersbergen, S. Kraft, Proc SPIE 5488, 829-836 (2004)

5. D. L. Windt Computers in Physics 12, 360-370 1998

6. W. Görner, M.P. Hentschel, B.R. Müller, H. Riesemeier, M. Krumrey, G. Ulm, W. Diete, U. Klein and R. Frahm Nucl. Instr. and Meth. A 467-468, 703 - 706 (2001)

7. E. Ziegler, J. Hoszowska, T. Bigault, L. Peverini, J. Y. Massonnat, and R. Hustache in AIP Conference Proceedings Volume 705 Synchrotron Radiation Iinstrumentation: Eighth International Conference on Synchrotron Radiation Instrumentation ed T Warwick et al . 436-439 (2004)

8. Novel multilayer design for future hard x-ray missions, F. E. Christensen, C. P. Jensen, K. K. Madsen, C. M. H. Chen, M. J. Pivovaroff, P. Hoghoj, A. Dariel, , Proc SPIE 6266 this volume

9. G.Pareschi and V.Cotroneo, Proc. SPIE 5168, 53-64 (2003)

10. Ziegler, J.F., SRIM, The Stopping and Range of Ions in Matter, v. 2000.35, http://www.research.ibm.com/ionbeams/SRIM/SRIMINTR.HTM 